\title{
Assessment of synchronous neural activities revealed by regional homogeneity in individuals with acute eye pain: a resting-state functional magnetic resonance imaging study
}

This article was published in the following Dove Press journal: Journal of Pain Research

\author{
Li-Yuan Tang ${ }^{1, *}$ \\ Hai-Jun $\mathrm{Li}^{2, *}$ \\ Xin Huang' \\ Jing Bao' \\ Zubin Sethi ${ }^{3}$ \\ Lei Ye' \\ Qing Yuan' \\ Pei-Wen Zhu' \\ Nan Jiang' \\ Gui-Ping Gao' \\ Yi Shao'
}

'Department of Ophthalmology, The First Affiliated Hospital of Nanchang University, Nanchang, Jiangxi, China; ${ }^{2}$ Department of Radiology, The First Affiliated Hospital of Nanchang University, Nanchang, Jiangxi, China; ${ }^{3}$ The Department of Medicine, University of Miami, Coral Gables, FL, USA

*These authors contributed equally to this work

Correspondence: Yi Shao; Gui-Ping Gao Department of Ophthalmology, The First Affiliated Hospital of Nanchang University, Number 17, Yongwaizheng Street, Donghu District, Nanchang 330006, Jiangxi, China

Tel +86 79l 88692520

Email freebee99@163.com;

ggpn@sina.com
Objective: Previous neuroimaging studies have demonstrated that pain-related diseases are associated with brain function and anatomical abnormalities, whereas altered synchronous neural activity in acute eye pain (EP) patients has not been investigated. The purpose of this study was to explore whether or not synchronous neural activity changes were measured with the regional homogeneity (ReHo) method in acute EP patients.

Methods: A total of 20 patients (15 males and 5 females) with EP and 20 healthy controls (HCs) consisting of 15 and 5 age-, sex-, and education-matched males and females, respectively, underwent resting-state functional magnetic resonance imaging. The ReHo method was applied to assess synchronous neural activity changes.

Results: Compared with HCs, acute EP patients had significantly lower ReHo values in the left precentral/postcentral gyrus (Brodmann area [BA]3/4), right precentral/postcentral gyrus (BA3/4), and left middle frontal gyrus (BA6). In contrast, higher ReHo values in acute EP patients were observed in the left superior frontal gyrus (BA11), right inferior parietal lobule (BA39/40), and left precuneus (BA7). However, no relationship was found between the mean ReHo signal values of the different areas and clinical manifestations, which included both the duration and degree of pain in EP patients.

Conclusion: Our study highlighted that acute EP patients showed altered synchronous neural activities in many brain regions, including somatosensory regions. These findings might provide useful information for exploration of the neural mechanisms underlying acute EP.

Keywords: acute eye pain, corneal pain, regional homogeneity, resting state, functional magnetic resonance imaging

\section{Introduction}

Eye pain (EP) is a common symptom in various eye diseases. Acute corneal pain is the main form of serious EP. The cornea is supplied by the ophthalmic branch of the trigeminal nerve and is one of the most densely innervated structures in the body. Thus, serious corneal pain usually occurs in keratitis and corneal ulcer patients. According to a survey from central China, the prevalence of infectious keratitis was $0.148 \%{ }^{1}$ Corneal pain is not only associated with eye symptoms, including photophobia and tears, but is also accompanied by headaches. ${ }^{2}$

In one study, corneal pain was shown to activate the trigemino-parabrachial pathway. ${ }^{3}$ A previous study in mice demonstrated that corneal pain could lead to neuronal activation in the spinal trigeminal nucleus, insular cortex, and anterior 
cingulated cortex. ${ }^{4}$ Meanwhile, a study involving neuroimaging research indicated that corneal pain in patients appeared to be associated with activation of the primary somatosensory cortex. ${ }^{5}$ Furthermore, EP has been frequently shown to be accompanied by mental disorders. Crane et al reported that the neuropathic EP patients demonstrated psychiatric problems/disease. ${ }^{6}$ A recent study highlighted that psychiatric or neurological disorders may occur in dry eye patients with neuropathic EP syndrome. ${ }^{7}$ Chronic EP in individuals was shown to be accompanied by depression. ${ }^{8}$ Moreover, two previous studies indicated that chronic pain may be linked to mental disorders. ${ }^{9}{ }^{10}$ However, there are only a few studies that have focused on synchronous neural activity changes in EP patients. Whether or not acute EP leads to the cerebral activity changes remains unknown.

Synchronous neuronal activity occurs in the normal human brain. ${ }^{11}$ Synchronous neuronal activity plays a critical role in learning and memory. ${ }^{12}$ In addition, reliable propagation of synchronous neuronal activity was shown to be crucial for neuronal information processing. ${ }^{13}$ Several previous electroencephalographic and functional magnetic resonance imaging (fMRI) studies indicated that synchronous neuronal activity might have a critical role in neurophysiological activity. ${ }^{14-16}$ Resting-state (rs)-fMRI is an effective method that has been successfully applied for changes in brain activity evaluations. The regional homogeneity (ReHo) method, a resting-state fMRI (rs-fMRI) measurement method, is thought to be a reliable and sensitive measurement, which can be used to evaluate coherence of the blood oxygen leveldependent (BOLD) signal among neighboring voxels of the whole brain at rest. ${ }^{17,18}$ The ReHo method is widely used to investigate the local synchronization of spontaneous fMRI signals. Moreover, the high test-retest reliability of ReHo has been confirmed by careful optimization of the data, preprocessing, and computational implementation. ReHo was developed to evaluate regional resting-state brain activity. ${ }^{19}$ Using the ReHo method, Yoshino et al found that distinct brain functions were related to depression and chronic pain. ${ }^{20}$ Another ReHo study demonstrated that persistent somatoform pain disorder was associated with dysfunction of brain activity in many regions. ${ }^{21}$ In our previous studies, the ReHo method was successfully used to assess synchronous neural activities in some eye diseases such as optic neuritis, ${ }^{22}$ comitant strabismus, ${ }^{23}$ and acute open-globe injury. ${ }^{24}$

Our study is the first to use the ReHo method for evaluating synchronous neural activity changes in acute EP patients. We hypothesized that acute EP might be associated with the altered pain-related brain regions.

\section{Participants and methods}

\section{Participants}

The research protocol was approved by the Medical Ethics Committee of the Ophthalmology Department of the First Affiliated Hospital of Nanchang University. All subjects participated voluntarily and provided written informed consent. All participants enrolled in the study met the following inclusion criteria: 1) were able to be scanned with an MRI (no cardiac pacemaker or implanted metal devices); 2) no cardiovascular system diseases such as heart disease and/or hypertension; 3) no psychiatric disorders (depression and/ or anxiety disorders); and 4) no cerebral infarction diseases (cerebral hemorrhage, cerebral infarction, and/or cerebral vascular malformations).

Twenty patients with acute EP (15 males and 5 females) participated in the study. The mean duration of acute EP was $27 \pm 6.21$ days (range 21-33 days). The diagnostic criteria of EP patients included two parameters: 1) acute EP patients with keratitis or corneal ulcers and 2) both eyes without any other ocular diseases (strabismus, amblyopia, cataracts, glaucoma, optic neuritis, and/or retinal degeneration).

The exclusion criteria of EP included the following parameters: 1) long-term, chronic (>3 months) EP; 2) EP due to ocular trauma; 3) EP with serious related complications (ocular perforation, endophthalmitis, and/or orbital cellulitis); 4) no painkillers prior to fMRI scanning; and/or 5) EP associated with headaches or pains in other parts of the body.

Twenty (15 males and 5 females) healthy controls (HCs) participated in the study and were matched to EP patients with respect to in age, sex, and education. All HCs met the two criteria: 1) without any ocular diseases (dry eye, strabismus, amblyopia, cataracts, glaucoma, optic neuritis, retinal degeneration, and others), and 2) naked eye corrected visual acuity $>1.0$.

\section{MRI parameters}

MRI scanning was performed with a 3-Tesla MR scanner (Trio, Siemens, Munich, Germany). The whole-brain T1-weights were obtained with a magnetization-prepared gradient echo image (MPRAGE) with certain parameters: 1) repetition time $=1,900 \mathrm{~ms} ; 2$ ) echo time $=2.26 \mathrm{~ms} ; 3$ ) thickness $=1.0 \mathrm{~mm}$; 4) gap $=0.5 \mathrm{~mm} ; 5$ ) acquisition matrix $=256 \times 256 ; 6$ ) field of view $=250 \times 250 \mathrm{~mm}$; and 7) flip angle $=9^{\circ}$. Functional images involved repetition time $=2,000$ $\mathrm{ms}$, echo time $=30 \mathrm{~ms}$, thickness $=4.0 \mathrm{~mm}$, gap $=1.2 \mathrm{~mm}$, acquisition matrix $=64 \times 64$, flip angle $=90^{\circ}$, and field of view $=220 \times 220 \mathrm{~mm}$. Twenty-nine axial slices covering the entire brain were corrected. 


\section{fMRI data processing}

The functional images were analyzed as describe previously. ${ }^{24}$ Briefly, the data were filtered by software (www.MRIcro.

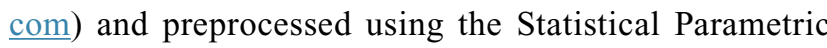
Mapping SPM8 (http://www.fil.ion.ucl.ac.uk/spm/) and Data Processing Assistant for rs-fMRI DPARSFA (http://rfmri.org) DPARSF) software..$^{25}$ The first 10 volumes of each subject were discarded due to the signal reaching equilibrium. The remaining 230 volumes of functional BOLD images were corrected for slice timing effects and motion. Individual T1-weighted MPRAGE structural images were registered to the mean fMRI data, and then the resulting aligned T1-weighted images were segmented using the diffeomorphic anatomical registration through the exponentiated Lie algebra toolbox for improving spatial precision in normalization of fMRI data. ${ }^{26}$ The fMRI images were detrended and band pass-filtered $(0.01-0.08 \mathrm{~Hz})$ in order to reduce the effects of low-frequency drift and physiological high-frequency respiratory and cardiac noise. ${ }^{27}$ Based on Kendall's coefficient of concordance, each brain voxel was calculated in a voxel-wise manner by applying a cluster size of 26 voxels. According to the protocol from a previous study, we did not regress the global signal out. ${ }^{28}$ Finally, the remaining data were smoothed with a Gaussian kernel of $4 \times 4 \times 4 \mathrm{~mm}^{3}$ fullwidth at half-maximum.

\section{ReHo statistical analysis}

The ReHo calculation was performed with REST (http:// www.restfmri.net) software. The ReHo was used to analyze rs-fMRI data. The indices of locally synchronous activity were measured by calculating the voxel-wise similarities in activity fluctuations over a given voxel time course. ReHo values indicated regionally localized temporal synchronization within the cluster by using different spatial scales that had a direct dependence on their neighbors' cluster sizes. ReHo calculations at rest were done as previously described. ${ }^{29}$ To exclude the effects of confounding covariates on ReHo values, age, sex, and total brain volume were included as covariates in the analysis. Covariates of age, sex, and brain volume were regressed out using the DPARSF software toolbox. ${ }^{25}$

\section{Statistical analysis}

The cumulative clinical measurements, including the duration of EP onset, were analyzed in the study with an independent sample $t$-test using SPSS version 16.0 (SPSS Inc, Chicago, IL, USA). $P<0.05$ was considered to indicate significant differences. The visual analog scale pain scores were obtained for all EP subjects.

Statistical analysis was performed using a general linear model with the SPM8 toolkit. ReHo maps were transformed to $z$-score maps. Areas with significant changes of frequency or overall ReHo were selected as regions of interest (ROIs). Paired $t$-tests were used to examine the differences in ReHo at different frequency bands in these ROIs between the EP groups and the HCs $(P<0.01)$ for multiple comparisons using Gaussian Random Field (GRF) theory $(z>2.3, P<0.01$, cluster $>40$ voxels, AlphaSim corrected). The mean ReHo values in the different brain regions between the two groups were analyzed using receiver operating characteristic (ROC) curves. The Pearson's correlation was used to evaluate the relationship between the mean ReHo values in different brain regions in the EP group versus HCs and also between behavioral performances in both groups.

\section{Results}

\section{Demographics and visual measurements}

We did not find any differences in weight $(P=0.845)$ or age $(P=0.796)$ between the two groups $(P>0.05)$. The mean \pm standard deviation of the EP duration was $27 \pm 6.21$ days (more details are given in Table 1).

\section{ReHo differences}

Compared with HCs, acute EP patients had significantly lower ReHo values in the left precentral/postcentral gyrus (Brodmann area $[\mathrm{BA}] 3 / 4$ ), right precentral/postcentral gyrus (BA3/4), and left middle frontal gyrus (BA6) as shown in Figure 1A and B (blue) and Table 2. Meanwhile, higher ReHo values in the EP patient group were observed in the left superior frontal gyrus (BA11), right inferior parietal lobule (BA39/40), and left precuneus (BA7) as shown in Figure 1A and $\mathrm{B}$ (red) and Table $2(P<0.01$ for multiple comparisons using the GRF theory, $z>2.3, P<0.01$, cluster $>40$ voxels,

Table I Demographics and clinical measurements by group

\begin{tabular}{lllll}
\hline Condition & EP & HC & t-value & P-value* \\
\hline Male/female & $15 / 5$ & $15 / 5$ & N/A & $>0.99$ \\
Age (years) & $51.62 \pm 5.20$ & $51.26 \pm 5.27$ & 0.260 & 0.796 \\
Weight (kg) & $63.56 \pm 7.12$ & $63.18 \pm 6.68$ & 0.197 & 0.845 \\
Handedness & $20 \mathrm{R}$ & $20 \mathrm{R}$ & $\mathrm{N} / \mathrm{A}$ & $>0.99$ \\
Duration of EP (days) & $27 \pm 6.2 \mathrm{I}$ & $\mathrm{N} / \mathrm{A}$ & $\mathrm{N} / \mathrm{A}$ & $\mathrm{N} / \mathrm{A}$ \\
VAS pain scores & $4.05 \pm 0 . .94$ & $\mathrm{~N} / \mathrm{A}$ & $\mathrm{N} / \mathrm{A}$ & $\mathrm{N} / \mathrm{A}$
\end{tabular}

Notes: $* P<0.05$, independent $t$-tests comparing two groups; the data are shown in mean \pm standard deviation.

Abbreviations: HC, healthy control; N/A, not applicable; EP, eye pain; VAS, visual analog scale. 


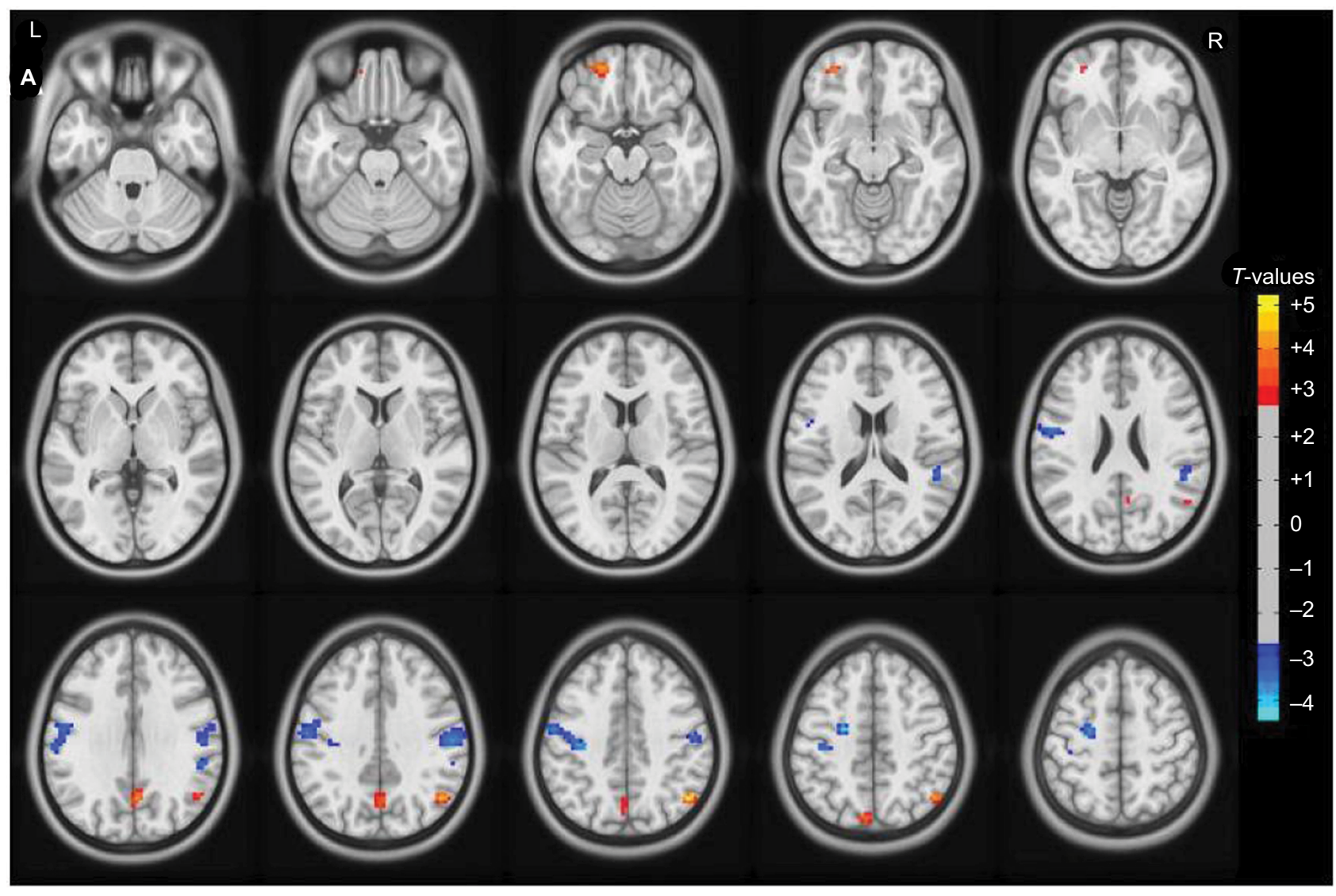

B
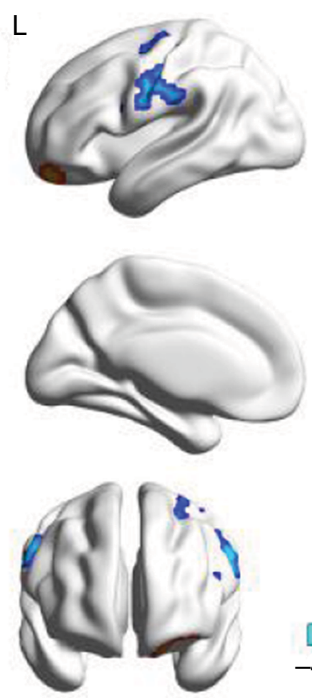
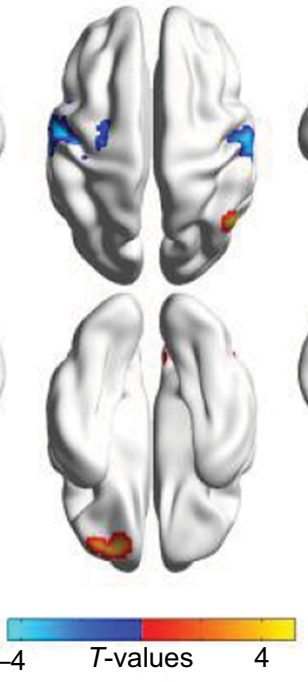

C

$\mathrm{R}$
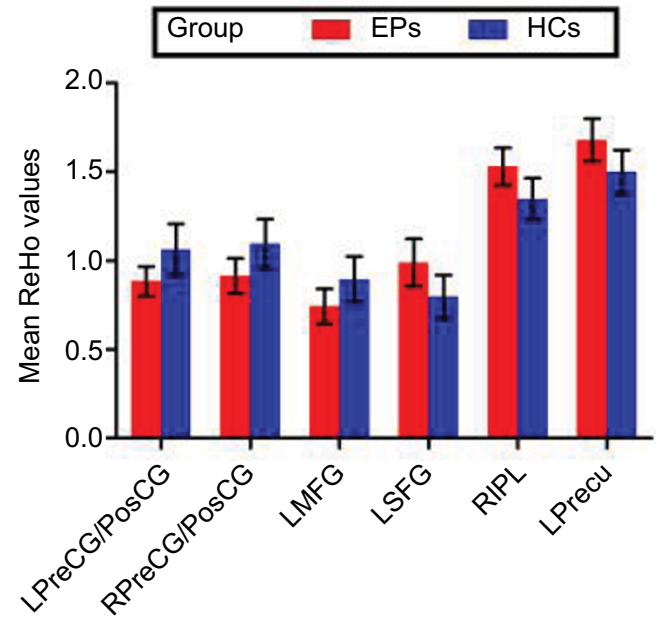

Altered ReHo regions

Figure I Spontaneous brain activity in the EPs versus HCs. (A and B) Significant activity differences were observed in the left precentral/postcentral gyrus (BA3/4), right precentral/postcentral gyrus (BA3/4), left middle frontal gyrus (BA6), left superior frontal gyrus (BAII), right inferior parietal lobule (BA39/40), and left precuneus (BA7). The red or yellow denotes higher ReHo values, and the blue areas indicate lower ReHo values $(P<0.01$ for multiple comparisons using Gaussian Random Field theory $(z>2.3$, $P<0.0$ I, cluster $>40$ voxels, AlphaSim corrected corrected). (C) The mean values of altered ReHo values between the EPs and $\mathrm{HC}$ groups.

Abbreviations: ReHo, regional homogeneity; EP, eye pain; HCs, healthy controls; PreCG/PosCG, precentral/postcentral gyrus; MFG, middle frontal gyrus; SFG, superior frontal gyrus; IPL, inferior parietal lobule; Precu, precuneus; L, left; R, right; BA, Brodmann area.

AlphaSim corrected). The mean values of altered ReHo values between the two groups are shown with a histogram (Figure 1C). In the EP group, we did not find any correlation between the mean ReHo values in different brain regions and clinical manifestations $(P>0.05)$.

\section{ROC curve}

We hypothesized that the differences in ReHo values between the EP and $\mathrm{HC}$ groups might be useful as diagnostic markers. The mean ReHo values in different brain regions were analyzed using the ROC method. The area under the ROC 
Table 2 Brain areas with significantly different ReHo values between groups

\begin{tabular}{|c|c|c|c|c|c|c|}
\hline \multirow[t]{2}{*}{ Brain regions/conditions } & \multirow[t]{2}{*}{ BA } & \multicolumn{3}{|c|}{ MNI coordinates } & \multirow[t]{2}{*}{ Cluster size } & \multirow[t]{2}{*}{ t-value } \\
\hline & & $\bar{x}$ & $\mathbf{Y}$ & $\mathbf{Z}$ & & \\
\hline \multicolumn{7}{|l|}{ Eye pain $<\mathrm{HCs}$} \\
\hline Left precentral/postcentral gyrus & $3 / 4$ & -39 & -24 & 45 & 187 & -3.803 \\
\hline Right precentral/postcentral gyrus & $3 / 4$ & 57 & -18 & 36 & 149 & -3.661 \\
\hline Left middle frontal gyrus & 6 & -24 & -9 & 48 & 49 & -4.452 \\
\hline \multicolumn{7}{|l|}{ Eye pain > HCs } \\
\hline Left superior frontal gyrus & 11 & -21 & 51 & -18 & 54 & 4.31 \\
\hline Right inferior parietal lobule & $39 / 40$ & 48 & -63 & 42 & 70 & 5.124 \\
\hline Left precuneus & 7 & 3 & -63 & 30 & 81 & 4.051 \\
\hline
\end{tabular}

Notes: The statistical threshold was set at the voxel level with $P<0.05$ for multiple comparisons using Gaussian Random Field theory $(z>2.3, P<0.01$, cluster $>40$ voxels, AlphaSim corrected).

Abbreviations: ReHo, regional homogeneity; BA, Brodmann area; HCs, healthy controls; MNI, Montreal Neurological Institute.

A

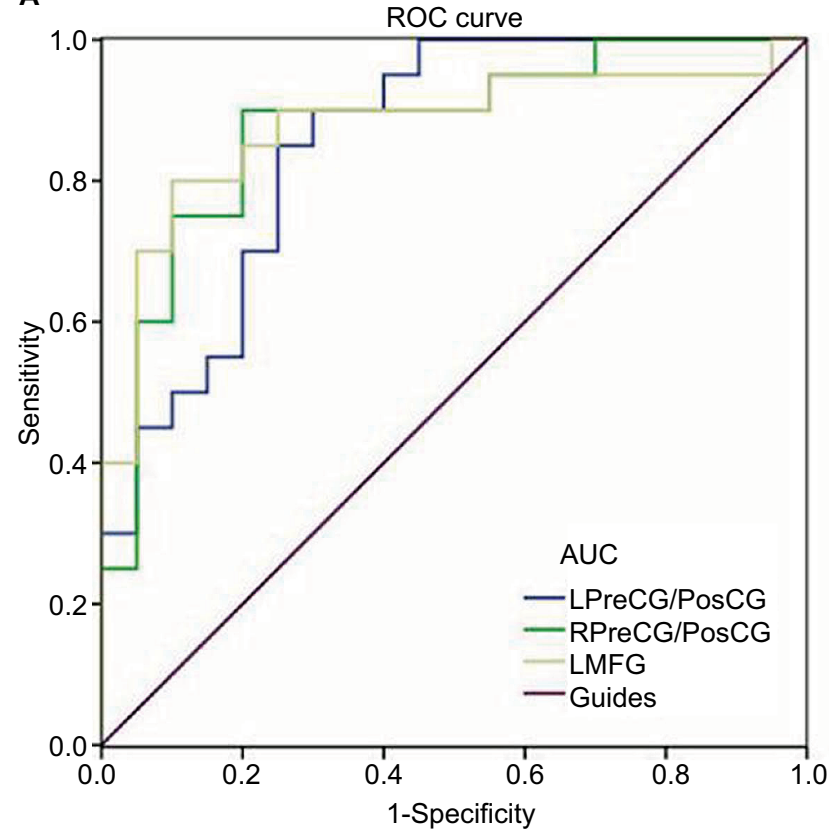

B

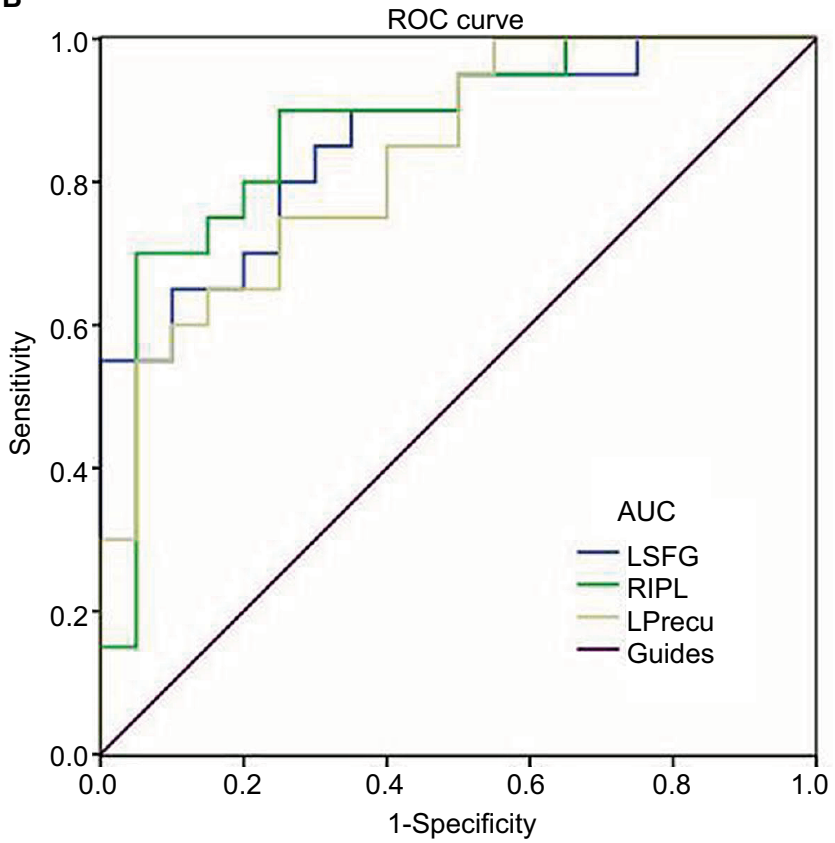

Figure 2 ROC curve analysis of the mean ReHo values for altered brain regions.

Notes: The areas under the ROC curve were (A) LPreCG/PosCG (0.873) ( $(P<0.001 ; 95 \%$ Cl: $0.739-0.971)$; RPreCG/PosCG $(0.875)(P<0.001$; $95 \%$ Cl: $0.763-0.987)$; LMFG (0.878) (P<0.00I; 95\%Cl: 0.76I-0.994), (EPs < HCs). (B) LSFG (0.860) (P<0.00I; 95\%Cl: 0.747-0.973) and RIPL $(0.873)(P<0.001$; $95 \% \mathrm{Cl}$ : $0.759-0.986)$; LPrecu $(0.832)$ ( $P<0.001 ; 95 \% \mathrm{Cl}: 0.710-0.955)$ (EPs $>\mathrm{HCs})$.

Abbreviations: AUC, area under the curve; ROC, receiver operating characteristic; ReHo, regional homogeneity; Cl, confidence interval; EP, eye pain; HCs, healthy controls; PreCG/PosCG, precentral/postcentral gyrus; MFG, middle frontal gyrus; SFG, superior frontal gyrus; IPL, inferior parietal lobule; Precu, precuneus; L, left; R, right.

curve stands for diagnostic rate. The area under the curve (AUC) values of 0.5-0.7 indicate low accuracy, AUC values of 0.7-0.9 indicate middle accuracy, and AUC values $>0.9$ indicate high accuracy. The areas under the ROC curve for ReHo values yielded the following values: left precentral/ postcentral gyrus 0.873 ; right precentral/postcentral gyrus 0.875; left middle frontal gyrus 0.878 (Figure 2A, EPs < $\mathrm{HCs}$ ); left superior frontal gyrus 0.860 ; right inferior parietal lobule 0.873; and left precuneus 0.832 (Figure 2B, EPs $>\mathrm{HCs}$.

\section{Discussion}

To our knowledge, this is the first study to investigate synchronous neural activity changes, measured by the ReHo method, in acute EP patients. In our study, EP patients had significantly lower ReHo values in the left precentral/postcentral gyrus (BA3/4), right precentral/postcentral gyrus (BA3/4), and left middle frontal gyrus (BA6). Nevertheless, the higher ReHo values in acute EP patients were observed in the left superior frontal gyrus (BA11), right inferior parietal lobule (BA39/40), and left precuneus (BA7). 
The postcentral gyrus, known as the primary somatosensory cortex (S1), plays a critical role in the sense of touch ${ }^{30}$ and social touch. ${ }^{31}$ In addition, S1 is involved in representation of pain. ${ }^{32,33}$ Previous neuroimaging studies have shown that many pain-related diseases may be involved in S1 dysfunction. Pijnenburg et al demonstrated that patients with low back pain showed decreased functional connectivity in the left precentral gyrus and lobule and left cerebellum when compared with HCs. ${ }^{34}$ Another study reported that traumatic spinal cord injury and pain symptoms in patients were associated with reduced gray matter (GM) volumes in their left secondary somatosensory cortex.${ }^{35}$ Previous studies demonstrated that the altered GM volume was linked to ReHo value changes. ${ }^{36,37} \mathrm{~A}$ recent study showed that rheumatoid arthritis patients with chronic pain exhibited increased brain connectivity in the supplementary motor areas. ${ }^{38}$ In support of these findings, we found that individuals with acute EP exhibited significantly lower ReHo values in the bilateral postcentral gyrus (BA3), indicating dysfunction of cerebral activities in postcentral gyrus. Therefore, we speculate that individuals with acute EP may have associated dysfunctional brain activity in the postcentral gyrus.

The precentral gyrus (BA4) is the site of the primary motor cortex (M1), which plays an important role in motor performance ${ }^{39}$ and muscle and movement control. ${ }^{39}$ Previous studies demonstrated that electrical stimulation of the precentral gyrus may be a good treatment for central pain..$^{40,41}$ Yoshino et al reported that somatoform pain showed activation in the precentral gyrus. ${ }^{42}$ In our study, we found that EP patients have significantly lower ReHo values in the bilateral precentral gyrus (BA4), which reflects M1 dysfunction. Thus, our results suggest that acute EP may lead to motor control dysfunction.

It is well known that the middle frontal gyrus plays an important role in cognitive and attention function. ${ }^{43}$ Several previous studies demonstrated that frontal gyrus dysfunction occurred in depressed patients. ${ }^{44-46}$ According to surveys in China, dry eyes and corneal symptoms in individuals appear to be associated with depression in these individuals. ${ }^{47,48} \mathrm{In}$ our study, we found that EP patients showed lower ReHo values in the left middle frontal gyrus (BA6), which reflects middle frontal gyrus dysfunction. Thus, we speculate that middle frontal gyrus dysfunction might be related to depression in EP patients.

The precuneus (BA7) is a part of the superior parietal lobule, which plays a critical role in coordination of motor skills ${ }^{49}$ and working memory. ${ }^{50}$ In addition, the precuneus is a core constituent of the default-mode network (DMN). ${ }^{51}$
The precuneus was shown to be related to pain sensitivity in healthy adults..$^{52}$ In our study, we demonstrated that the acute EP patients show increased ReHo values in the left precuneus, which indicates left precuneus dysfunction. We speculate that acute EP might lead to the dysfunction of the DMN.

\section{Conclusion}

In summary, our results revealed that acute EP patients showed altered synchronous neural activities in many brain regions, including somatosensory regions. These findings might provide useful information for exploration of neural mechanisms in acute EP patients.

\section{Acknowledgments}

This work was supported by grants from the National Natural Science Foundation of China (numbers 81660158, 81460092, 81400372, and 81400424), Natural Science Key Project of Jiangxi Province (number 20161ACB21017), Youth Science Foundation of Jiangxi Province (number 20151BAB215016), Key Research Development Foundation of Jiangxi Province (number 20151BBG70223), Key Education Department Foundation of Jiangxi Province (number GJJ160020), Education Department Foundation of Jiangxi Province (number GJJ160122), and Health Development Planning Commission Science Foundation of Jiangxi Province (numbers 20164017 , 20175115, and 20175116).

\section{Disclosure}

This was not an industry-supported study. The authors report no conflicts of interest in this work.

\section{References}

1. Cao J, Yang Y, Yang W, et al. Prevalence of infectious keratitis in Central China. BMC Ophthalmol. 2014;14(1):43.

2. Sandrini G, Alfonsi E, Ruiz L, et al. Impairment of corneal pain perception in cluster headache. Pain. 1991;47(3):299-304.

3. Aicher SA, Hegarty DM, Hermes SM. Corneal pain activates a trigemino-parabrachial pathway in rats. Brain Res. 2014;1550(11):18-26.

4. Xiang Y, Zhou W, Wang P, et al. Alkali burn induced corneal spontaneous pain and activated neuropathic pain matrix in the central nervous system in mice. Cornea. 2017;36(11):1408-1414.

5. Moulton EA, Becerra L, Rosenthal P, Borsook D. An approach to localizing corneal pain representation in human primary somatosensory cortex. PLoS One. 2012;7(9):e44643.

6. Crane AM, Levitt RC, Felix ER, Sarantopoulos KD, McClellan AL, Galor A. Patients with more severe symptoms of neuropathic ocular pain report more frequent and severe chronic overlapping pain conditions and psychiatric disease. Br J Ophthalmol. 2017;101(2): 227-231.

7. Han SB, Yang HK, Hyon JY, Wee WR. Association of dry eye disease with psychiatric or neurological disorders in elderly patients. Clin Interv Aging. 2017;12:785-792.

8. Naushad N, Dunn LB, Muñoz RF, et al. Depression increases subjective stigma of chronic pain. J Affect Disord. 2018;229:456-462. 
9. Benjamin S, Morris S, McBeth J, et al.The association between chronic widespread pain and mental disorder: a population-based study. Arthritis Rheum. 2000;43(3):561-567.

10. Braden JB, Zhang L, Zimmerman FJ, et al. Employment outcomes of persons with a mental disorder and comorbid chronic pain. Psychiatr Serv. 2008;59(8):878-885.

11. Sturm AK, König P. Mechanisms to synchronize neuronal activity. Biol Cybern. 2001;84(3):153-172.

12. Jutras MJ, Buffalo EA. Synchronous neural activity and memory formation. Curr Opin Neurobiol. 2010;20(2):150-155.

13. Bayati M, Valizadeh A, Abbassian A, et al. Self-organization of synchronous activity propagation in neuronal networks driven by local excitation. Front Comput Neurosci. 2015;9(3):69.

14. Spencer KM, Nestor PG, Perlmutter R, et al. Neural synchrony indexes disordered perception and cognition in schizophrenia. Proc Natl Acad Sci US A. 2004;101(49):17288-17293.

15. Ward LM. Synchronous neural oscillations and cognitive processes. Trends Cogn Sci. 2003;7(12):553-559.

16. Li R, Li Y, An D, Gong Q, Zhou D, Chen H. Altered regional activity and inter-regional functional connectivity in psychogenic non-epileptic seizures. Sci Rep. 2015;5:11635.

17. Zang Y, Jiang T, Lu Y, et al. Regional homogeneity approach to fMRI data analysis. Neuroimage. 2004;22(1):394-400.

18. Tononi G, McIntosh AR, Russell DP, et al. Functional clustering: identifying strongly interactive brain regions in neuroimaging data. Neuroimage. 1998;7(2):133-149.

19. Zuo XN, Xu T, Jiang L, et al. Toward reliable characterization of functional homogeneity in the human brain: preprocessing, scan duration, imaging resolution and computational space. Neuroimage. 2013; 65:374-386.

20. Yoshino A, Okamoto Y, Doi M, et al. Regional brain functions in the resting state indicative of potential differences between depression and chronic pain. Sci Rep. 2017;7(1):3003.

21. Huang T, Zhao Z, Yan C, et al. Altered spontaneous activity in patients with persistent somatoform pain disorder revealed by regional homogeneity. PLoS One. 2016;11(3):e0151360.

22. Shao Y, Cai FQ, Zhong YL, et al. Altered intrinsic regional spontaneous brain activity in patients with optic neuritis: a resting-state functional magnetic resonance imaging study. Neuropsychiatr Dis Treat. 2015;11:3065-3073.

23. Huang X, Li SH, Zhou FQ, et al. Altered intrinsic regional brain spontaneous activity in patients with comitant strabismus: a resting-state functional MRI study. Neuropsychiatr Dis Treat. 2016;12:1303-1308.

24. Huang X, Li HJ, Ye L, et al. Altered regional homogeneity in patients with unilateral acute open-globe injury: a resting-state functional MRI study. Neuropsychiatr Dis Treat. 2016;12:1901-1906.

25. Chao-Gan Y, Yu-Feng Z. DPARSF: a MATLAB toolbox for "pipeline" data analysis of resting-state fMRI. Front Syst Neurosci. 2010;4:13.

26. Goto M, Abe O, Aoki S, et al. Diffeomorphic anatomical registration through exponentiated Lie algebra provides reduced effect of scanner for cortex volumetry with atlas-based method in healthy subjects. Neuroradiology. 2013;55(7):869-875.

27. Lowe MJ, Mock BJ, Sorenson JA. Functional connectivity in single and multislice echoplanar imaging using resting-state fluctuations. Neuroimage. 1998;7(2):119-132.

28. Murphy K, Birn RM, Handwerker DA, et al. The impact of global signal regression on resting state correlations: are anti-correlated networks introduced? Neuroimage. 2009;44(3):893-905.

29. Zang Y, Jiang T, Lu Y, et al. Regional homogeneity approach to fMRI data analysis. Neuroimage. 2004;22(1):394-400.

30. Pihko E, Nangini C, Jousmäki V, Hari R. Observing touch activates human primary somatosensory cortex. Eur JNeurosci. 2010;31(10):1836-1843.

31. Gazzola V, Spezio ML, Etzel JA, Castelli F, Adolphs R, Keysers C. Primary somatosensory cortex discriminates affective significance in social touch. Proc Natl Acad Sci USA. 2012;109(25):E1657-E1666.
32. Ploner M, Schmitz F, Freund HJ, Schnitzler A. Differential organization of touch and pain in human primary somatosensory cortex. J Neurophysiol. 2000;83(3):1770-1776.

33. Frot M, Magnin M, Mauguière F, Garcia-Larrea L. Cortical representation of pain in primary sensory-motor areas (S1/M1) - a study using intracortical recordings in humans. Hum Brain Mapp. 2013;34(10):2655-2668.

34. Pijnenburg M, Brumagne S, Caeyenberghs K, et al. Resting-state functional connectivity of the sensorimotor network in individuals with nonspecific low back pain and the association with the sit-to-stand-to-sit task. Brain Connect. 2015;5(5):303-311.

35. Jutzeler CR, Huber E, Callaghan MF, et al. Association of pain and CNS structural changes after spinal cord injury. Sci Rep. 2016;6(1):18534.

36. Lv SY, Zou QH, Cui JL, et al. Decreased gray matter concentration and local synchronization of spontaneous activity in the motor cortex in Duchenne muscular dystrophy. AJNR Am J Neuroradiol. 2011;32(11):2196-2200.

37. Borroni B, Premi E, Formenti A, et al. Structural and functional imaging study in dementia with Lewy bodies and Parkinson's disease dementia. Parkinsonism Relat Disord. 2015;21(9):1049-1055.

38. Karni A1, Meyer G, Rey-Hipolito C, et al. The acquisition of skilled motor performance: fast and slow experience-driven changes in primary motor cortex. Proc Natl Acad Sci USA. 1998;95(3):861-868.

39. Kakei S, Hoffman DS, Strick PL. Muscle and movement representations in the primary motor cortex. Science. 1999;285(5436):2136-2139.

40. Peyron R, Garcia-Larrea L, Deiber MP, et al. Electrical stimulation of precentral cortical area in the treatment of central pain: electrophysiological and PET study. Pain.1995;62(3):275-286.

41. Hsieh JC, Meyerson BA, Ingvar M. PET study on central processing of pain in trigeminal neuropathy. Eur J Pain.1999;3(1):51-65.

42. Yoshino A, Okamoto Y, Kunisato Y, et al. Distinctive spontaneous regional neural activity in patients with somatoform pain disorder: a preliminary resting-state fMRI study. Psychiatry Res. 2014;221(3):246-248

43. Tops M, Boksem MA. A potential role of the inferior frontal gyrus and anterior insula in cognitive control, brain rhythms, and event-related potentials. Front Psychol. 2011;2(4):330.

44. Andersson M, Ystad M, Lundervold A, Lundervold AJ. Correlations between measures of executive attention and cortical thickness of left posterior middle frontal gyrus - a dichotic listening study. Behav Brain Funct. 2009;5(1):41.

45. Chang CC, Yu SC, McQuoid DR, et al. Reduction of dorsolateral prefrontal cortex gray matter in late-life depression. Psychiatry Res. 2011;193(1):1-6.

46. Penfold C, Vizueta N, Townsend JD, Bookheimer SY, Altshuler LL. Frontal lobe hypoactivation in medication-free adults with bipolar II depression during response inhibition. Psychiatry Res. 2015;231(3):202-209.

47. Wen $\mathrm{W}, \mathrm{Wu} Y$, Chen Y, et al. Dry eye disease in patients with depressive and anxiety disorders in Shanghai. Cornea. 2012;31(6):686-692.

48. Labbé A, Wang YX, Jie Y, Baudouin C, Jonas JB, Xu L. Dry eye disease, dry eye symptoms and depression: the Beijing Eye Study. Br J Ophthalmol. 2013;97(11):1399-1403.

49. Wenderoth N, Debaere F, Sunaert S, SP Swinnen. The role of anterior cingulate cortex and precuneus in the coordination of motor behaviour. Eur J Neurosci. 2005;22(1):235-246.

50. Wallentin M, Weed E, Østergaard L, Mouridsen K, Roepstorff A. Accessing the mental space-spatial working memory processes for language and vision overlap in precuneus. Hum Brain Mapp. 2008;29(5):524-532.

51. Utevsky AV, Smith DV, Huettel SA. Precuneus is a functional core of the default-mode network. J Neurosci. 2014;34(3):932-940.

52. Goffaux P, Girard-Tremblay L, Marchand S, Daigle K, Whittingstall K. Individual differences in pain sensitivity vary as a function of precuneus reactivity. Brain Topogr. 2014;27(3):366-374. 
The Journal of Pain Research is an international, peer reviewed, open access, online journal that welcomes laboratory and clinical findings in the fields of pain research and the prevention and management of pain. Original research, reviews, symposium reports, hypothesis formation and commentaries are all considered for publication.
The manuscript management system is completely online and includes a very quick and fair peer-review system, which is all easy to use. Visit http://www.dovepress.com/testimonials.php to read real quotes from published authors. 